\title{
Inversion of Randomly Corrugated Surfaces Structure from Atom Scattering Data
}

\author{
Daniel A. Lidar \\ Chemistry Department \\ University of California \\ Berkeley, CA 94720 \\ $U S A$
}

\begin{abstract}
The Sudden Approximation is applied to invert structural data on randomly corrugated surfaces from inert atom scattering intensities. Several expressions relating experimental observables to surface statistical features are derived. The results suggest that atom (and in particular He) scattering can be used profitably to study hitherto unexplored forms of complex surface disorder.
\end{abstract}

\section{INTRODUCTION}

Structurally disordered surfaces have been a subject of great interest for some time now. Of special interest are epitaxially grown films, liquid surfaces, and amorphous surfaces. In epitaxial growth for example, metal or semiconductor atoms are adsorbed on a surface under thermal conditions, to form two- and three-dimensional structures on top of it. The physical and chemical properties are determined by the final form of these structures. These may be of dramatic importance, e.g, in the production of electronic devices. One of the most exciting aspects of epitaxial growth kinetics, is that it prepares disordered structures in the intermediate stages. The disorder manifests itself in the formation of various types of clusters or diffusion limited aggregates on top of the surface. These structures may be monolayers (usually at high temperatures, when the diffusivity is large, or at coverages significantly below a monolayer), in which case the disorder is two-dimensional, or they may be composed of several layers, giving rise to disorder in three dimensions. Epitaxially grown structures of this type offer an exceptional opportunity for both experimental and theoretical study of disorder. No satisfactory and comprehensive theory of the epitaxial growth process is as of yet available, much due to the absence of reliable interaction potentials for the system. The situation with respect to liquid and amorphous surfaces is similar: very little is known at this point about their structure. Progress at this stage thus hinges critically on data available from experiments. An important experimental technique is thermal atom scattering, and in particular He scattering [1 3]. The main advantage offered by He scattering is complete surface sensitivity, as He does not penetrate into the bulk, unlike other scattering techniques such as neutron or X-ray scattering, or low energy electron diffraction (LEED). Another important advantage is that He scattering is highly non-intrusive, due to the inertness and low mass of the He atoms. The latter also means that He scattering is really a diffraction experiment at the typical meV energy scale at which most experiments are performed, with sensitivity to atomic-scale features. The interpretation of He scattering experiments is, however, rather involved due to the complicated interaction between the He beam and the surface. As in all other scattering problems, this interpretation issue is in fact one of inversion of the He/surface potential. The inversion problem, however, is intrinsically ill-posed, since one can only measure intensities, not phases. There is a certain redundancy in the intensity information which can be exploited to obtain relative phase shifts, but never the absolute phases. Thus the inversion can never be fully performed, although partial information can be obtained, or useful approximations can be made concerning the shape of the potential, which yield an analytically closed-form solution $\left[\begin{array}{|l}6 \\ 6\end{array}\right.$.

In this work I will concentrate on the problem of trying to relate surface structural features to the scattering intensities, rather than the potential in general. This involves a non-trivial step of connecting the potential with such features. One possibility is the suggestion by Norskov and coworkers [7] on the basis of effective-medium theory, in which the leading repulsive term in the He scattering potential from any electronic system is taken to be proportional to the local unperturbed electron density $n_{0}(\mathbf{r})$ of the host at the He position $\mathbf{r}$ :

$$
V(\mathbf{r})=\alpha n_{0}(\mathbf{r}) .
$$

In this way the potential is rather simply related to structural features such as the local electronic corrugation. However, while useful for describing the interaction close to the surface, where repulsion dominates, this formula is inapplicable at large distances where the He/surface interaction is dominated by the long-range attractive forces. In addition, it still requires knowledge of the local electron density, often a highly nontrivial task, especially in presence of defects or impurities. Thus in practice one often resorts to the use of specific functional forms for the potential, such as the Morse or Lennard-Jones potentials, and fits the parameters to the experimental data. Unfortunately, the connection to surface geometrical features is then less transparent. 
So far almost all of the work done on inverting He/surface potentials has been for He scattering from ordered, crystalline surfaces. Again, a full inversion is impossible, but useful results have been obtained by assuming specific forms for the interaction potential. These include semiconductor surfaces: GaAs(110), $\mathrm{Si}, \operatorname{InSb}(110)$ [8], and some transition metal surfaces $(\mathrm{Ni}, \mathrm{Cu}, \mathrm{Ag}, \mathrm{Au})$ [9]. More recent efforts have concentrated on potentials for scattering from various adsorbed monolayers on metals, e.g., the $\mathrm{c}(2 * 2)$ phase of oxygen on $\mathrm{Ni}(001)$ [10], hydrogen-plated $\mathrm{Pt}$ [11], and $\mathrm{Xe} / \mathrm{Cu}(110)$ [12]. We recently fitted cross-section data to obtain a potential for a disordered $\mathrm{Ag} / \mathrm{Pt}(111)$ system [13]. A very detailed recent review of potentials of physical adsorption is the work of Vidali et al. [14], which tabulates parameters of interest as deduced from analysis of experimental data and calculations of over 250 gas-surface systems, including He.

Formal inversion methods have also occupied the attention of various researchers. The first such work was presented by Gerber and Yinnon [ब], who showed that atom-surface interaction potentials can be recovered from the diffraction peak intensities measured in beam scattering experiments by a direct, simple inversion method, using the Sudden approximation (SA). The SA is a highly successful and useful theoretical method in the He scattering field, and has been reviewed by Gerber [2]. The method of Ref. [4] was applied to simulated Ne scattering from W(110). This was followed by the first inversion of real atom/surface scattering data: the $\mathrm{He} / \mathrm{MgO}(100)$ system [15]. Schlup [16] showed how the surface profile function can be inverted in the Eikonal approximation, and applied his method to simulated data. In Ref. [6] we studied a related problem: the inversion of an ad-atom profile function in the SA. Rabitz and coworkers developed a method based on functional sensitivity analysis and Tikhonov regularization [17, and applied this to the inversion of the He potential for scattering from a Xe monolayer on the (0001) face of graphite [5, 18], which was also attempted earlier by employing close-coupling calculations [19]. Finally, we recently inverted the structure of a low temperature disordered overlayer of $\mathrm{Ag}$ on $\mathrm{Pt}(111)$ [20].

It should be noted that the situation with regards to potentials for He scattering from disordered surfaces is very much inferior to the case of ordered surfaces described above. Essentially no such reliable potentials exist, and the subject is at this point at a most preliminary stage. The common approach it to assume that the potential can be represented as a surface term plus a sum of pairwise additive terms representing the interaction of the $\mathrm{He}$ with each of the surface adatoms or vacancies. In this paper I will take a different approach, and will show how one can derive statistical information about randomly corrugated surfaces from He scattering measurements. This information comes in the form of correlation functions, not potential parameters. The resulting expressions relate useful statistical parameters characterizing the surface disorder to experimental observables which are not hard to obtain in practice, such as incidence energy dependence of the specular intensity. Unfortunately, at the time of writing experiments are unavailable for comparison with the results obtained here. The developments will therefore be primarily methodological, in anticipation of experimental data. It is hoped that the results obtained here will motivate He scattering experiments on disordered solid and liquid surfaces. As demonstrated by this work and others before it, He scattering can provide a wealth of information on disordered surface structure and dynamics.

The structure of the paper is as follows. Sec. II provides a brief introduction into the SA. Sec. III is the heart of the paper and derives the inversion expressions. Concluding remarks are brought in Sec.IV.

\section{BRIEF REVIEW OF THE SUDDEN APPROXIMATION}

Consider a He atom with mass $\mu$ incident upon a surface with wavevector $\mathbf{k}=\left(\mathbf{K}, k_{z}\right) . \hbar \mathbf{K}=\hbar\left(k_{x}, k_{y}\right)$ and $\hbar \mathbf{K}^{\prime}$ are respectively the intial and final momentum components parallel to the surface, and $\hbar k_{z}$ is the incident momentum normal to the surface. The position of the He atom is $\mathbf{r}=(\mathbf{R}, z)$, where $\mathbf{R}=(x, y)$ is the lateral position. The SA is valid when the collisional momentum transfer $\mathbf{q}=\mathbf{K}^{\prime}-\mathbf{K}$ in the direction parallel to the surface is much smaller than the momentum transfer normal to the surface: $2 k_{z} \gg|\mathbf{q}|$. This condition is satisfied at relatively high incidence angle and energy $E=(\hbar \mathbf{k})^{2} /(2 m)$, and moderate surface corrugations. When it holds, one can approximately consider the scattering along $z$ as occurring at fixed $\mathbf{R}$. Then if $\psi$ is the He wavefunction, it satisfies a Schrödinger equation where the dependence on $\mathbf{R}$ is adiabatic:

$$
\left[-\frac{\hbar^{2}}{2 \mu} \frac{d^{2}}{d z^{2}}+V_{\mathbf{R}}(z)\right] \psi_{\mathbf{R}}(z)=\varepsilon \psi_{\mathbf{R}}(z)
$$

Here $V_{\mathbf{R}}(z)$ is the He-surface interaction potential and no inelastic channels are included, so that the total energy $\varepsilon$ is conserved. This means that each surface point $\mathbf{R}$ gives rise to an elastic real phase shift $\eta(\mathbf{R})$, which can be evaluated in the WKB approximation from Eq.(2) as: 


$$
\eta(\mathbf{R})=\int_{\xi(\mathbf{R})}^{\infty} d z\left[\left(k_{z}{ }^{2}-\frac{2 m}{\hbar^{2}} V_{\mathbf{R}}(z)\right)^{1 / 2}-k_{z}\right]-k_{z} \xi(\mathbf{R}),
$$

where $\xi(\mathbf{R})$ is the classical turning point pertaining to the integrand in Eq.(3). The phase shift in turn yields the S-matrix as: $\mathcal{S}(\mathbf{R})=\exp [2 i \eta(\mathbf{R})]$. The $\mathbf{R}$ coordinate is conserved in this picture so the S-matrix is diagonal in the coordinate representation:

$$
\left\langle\mathbf{R}^{\prime}|\mathcal{S}| \mathbf{R}\right\rangle=e^{2 i \eta(\mathbf{R})} \delta\left(\mathbf{R}^{\prime}-\mathbf{R}\right) .
$$

Experimentally one measures probabilities $\left|\mathcal{S}\left(\mathbf{K}^{\prime} \rightarrow \mathbf{K}\right)\right|^{2}$ for $\mathbf{K} \rightarrow \mathbf{K}^{\prime}$ transitions. To obtain these $\langle\mathbf{R} \mid \mathbf{K}\rangle=\exp (i \mathbf{K}$. $\mathbf{R}) / \sqrt{A}$ (where $A$ is the area of the surface) can be used, to find:

$$
\left\langle\mathbf{K}^{\prime}|\mathcal{S}| \mathbf{K}\right\rangle=\int d \mathbf{R}^{\prime} d \mathbf{R}\left\langle\mathbf{K}^{\prime} \mid \mathbf{R}^{\prime}\right\rangle\left\langle\mathbf{R}^{\prime}|\mathcal{S}| \mathbf{R}\right\rangle\langle\mathbf{R} \mid \mathbf{K}\rangle=\frac{1}{A} \int d \mathbf{R} e^{-i \mathbf{q} \cdot \mathbf{R}} e^{2 i \eta(\mathbf{R})} .
$$

This is the well-known expression for the SA scattering amplitude [21]. The SA has been tested extensively by comparison to exact coupled-channel calculations on $\mathrm{Ne} / \mathrm{W}(110)$ and $\mathrm{He} / \mathrm{LiF}(001)$ [22], as well as by comparison to exact time-dependent propagation methods in the case of scattering from defects [23]. It is particularly noteworthy that the SA yielding an inverted potential of remarkable accuracy [4] for simulated Ne/W(110) data. The most important deficiency of the SA (apart from being limited to high energies) is its inability to describe double collision events. This is because, as mentioned above, the $\mathbf{R}$ coordinate is conserved in the SA, i.e., each trajectory takes place at constant R. Clearly, no double collisions can occur under such conditions. However, double collisions may take place, e.g., when an incident atom is scattered off a defect onto the surface, or in the opposite order. This issue was resolved recently by combining the SA with the Born approximation 24].

\section{INVERSION OF STRUCTURE OF RANDOMLY CORRUGATED SURFACES FROM He SCATTERING INTENSITIES}

\section{A. Atom Scattering from a Randomly Corrugated Square Well Potential}

Recall the role of $\xi(\mathbf{R})$ in the SA amplitude [Eq. (4) ]: it is the position of the classical turning points, which can alternatively be viewed as the surface corrugation function. In the case of a disordered surface $\xi(\mathbf{R})$ can be a random function of $\mathbf{R}$ and in order to obtain observable quantities one must average over an appropriate ensemble which characterizes the physical and statistical properties of the surface of interest. Thus the scattering probability, as a function of momentum transfer $\mathbf{Q}$, is given by

$$
P(\mathbf{Q})=\left\langle|S(\mathbf{Q})|^{2}\right\rangle,
$$

where $\langle\cdots\rangle$ indicates an average over the ensemble of which $\xi(\mathbf{R})$ is a typical sample. In certain cases one will be justified in assuming that translational invariance has been established after averaging over the corrugation ensemble. For example, this will generally be the case for liquid surfaces, for solids when the structural disorder on the surface is due to radiation damage, and epitaxially grown defects on a surface. This means that $\xi(\mathbf{R})$ is a stationary stochastic process, and hence that $\langle f[\xi(\mathbf{R})]\rangle$ is independent of $\mathbf{R}$, and that $\left\langle g\left[\xi(\mathbf{R}), \xi\left(\mathbf{R}^{\prime}\right)\right]\right\rangle$ depends only on $\mathbf{R}-\mathbf{R}^{\prime}$. The assumption of stationarity will, however, not hold for the case in which the structural disorder is caused by an incomplete adsorbed overlayer on a periodic substrate, and I will present a different treatment for such cases in a later section. Combining Eqs.(位, (5) one obtains in the case of a translationally invariant potential

$$
P(\mathbf{Q})=\frac{1}{A} \int d \mathbf{R} e^{i \mathbf{Q} \cdot \mathbf{R}}\left\langle e^{2 i[\eta(\mathbf{R})-\eta(0)]}\right\rangle .
$$

From the above expression it can be seen that within the SA, the angular scattering intensity is the Fourier transform of a function of the random variable

$$
E(\mathbf{R})=2[\eta(0)-\eta(\mathbf{R})]
$$

To proceed, one must first establish the connection between the phase-shift and properties of the surface. Assume now that the interaction of the He with the entire surface can be expressed in the form of a square well potential of 
depth $\epsilon$. Square well potentials have been rather successful in predicting properties of interacting gases; the accuracy obtained in fitting second and third virial coefficients with a square well potential compares to that of a Lennard-Jones 6-12 potential [25]. They have also been studied by others in the context of inversion problems [26,27, e.g., in neutron reflectometry from magnetic films 28. In our case the potential assumes the form:

$$
\frac{2 m}{\hbar^{2}} V(\mathbf{R}, z)= \begin{cases}\infty & : z<\xi(\mathbf{R}) \\ -\epsilon & : \xi(\mathbf{R})<z<\xi(\mathbf{R})+\Delta \xi(\mathbf{R})=\zeta(\mathbf{R}) \\ 0 & : z>\zeta(\mathbf{R})\end{cases}
$$

Typically, $\xi(\mathbf{R})$ is a relatively strongly corrugated function as it expresses the interaction of the He atom with the core electrons responsible for the steep repulsive part of the potential. On the other hand, $\zeta(\mathbf{R})$ may be very smooth, reflecting the loss of detail at the long distances at which the attractive part of the He-surface potential becomes important. According to Eq.(3) the phase shift is then given by

$$
\eta(\mathbf{R})=k_{z} \Delta \xi(\mathbf{R})\left((1+\delta)^{\frac{1}{2}}-1\right)-k_{z} \xi(\mathbf{R})
$$

where $\delta=\epsilon / k_{z}^{2}$ is a small parameter in the high-energy SA. Eq.(8) is known as "the Beeby effect" [29], i.e., in the presence of a well the wave number has to be replaced by an effective wave number which is due to the acceleration of the particle by the attractive forces. In the case of scattering by a pure hard wall $[\Delta \xi(\mathbf{R})=0]$, or a square well with the same shape as the hard wall $\left[\Delta \xi(\mathbf{R})=z_{0}=\right.$ constant], the scattering intensity is

$$
P(\mathbf{Q})=\frac{1}{A} \int d \mathbf{R} e^{i \mathbf{Q} \cdot \mathbf{R}}\left\langle e^{2 i k_{z}[\xi(0)-\xi(\mathbf{R})]}\right\rangle .
$$

In this case the intensity is essentially the Fourier transform of the characteristic function $\exp \left[i k_{z} Z(\mathbf{R})\right]$ of the $\operatorname{relative}$ surface corrugation:

$$
Z(\mathbf{R})=2[\xi(0)-\xi(\mathbf{R})]
$$

which makes its interpretation particularly clear. Knowledge of the probability density $f_{Z}(z ; \mathbf{R})$ fully determines the corrugation of the surface in question, in that $f_{Z}(z ; \mathbf{R}) d z$ is the probability of finding a corrugation of height between $z$ and $z+d z$ at $\mathbf{R}$, with respect to that at the origin. It is a well known fact in probability theory [30 that for any (piecewise-) continuous distribution function $f_{X}(x)$ a unique and explicit inversion of $f_{X}(x)$ exists from the characteristic function. Hence it is clear at the outset that He scattering can, at least in principle, provide very useful information about the statistics of a disordered system. In the following sections I will show how the statistical information pertaining to the disordered surface can be extracted from the He scattering intensity.

\section{B. Extraction of the Surface Corrugation Probability Density from the Angular Scattering Intensity for a Hard Wall Potential}

In this section I specialize to the hard wall potential. This model has been in use for many years in He scattering theory 31 33. and is related to the Eikonal approximation in optics 34. In Ref. 32] the origin of the hard-wall was discussed and it was concluded that it is due to the the local density of metal electron states in the selvedge. This leads to the Esbjerg-Norskov theory, Eq.(1). The great merits of the hard wall approximation are that (1) it is analytically tractable and (2) it provides a direct geometrical interpretation of the surface corrugation. However, it is clearly oversimplified and leaves out many interesting features of the He-surface interaction. More sophisticated approximation schemes have therefore been introduced by various researchers. The distorted-wave Born approximation was used early on [35] to treat soft potentials [36] and low-energy scattering [37. These studies have shown significant deviations from the predictions of the hard-wall model, e.g., for a corrugated Morse potential for He scattering by a $\mathrm{Cu}(110)$ surface [37. Nevertheless, the hard wall approximation has physical merit in the He high-energy limit, which is assumed here in connection with the SA.

An inverse Fourier transform of the scattering intensity $P(\mathbf{Q})$ [Eq.(9)] yields the characteristic function of the surface corrugation:

$$
\left\langle e^{i k_{z} Z(\mathbf{R})}\right\rangle=\frac{A}{2 \pi} \mathcal{F}_{k_{z}}^{-1}[P(\mathbf{Q}) ; \mathbf{R}]
$$


where a notation for the Fourier transform was introduced:

$$
\mathcal{F}[f(x) ; y]=\frac{1}{\sqrt{2 \pi}} \int_{-\infty}^{\infty} d x f(x) e^{i x y} .
$$

In the case of normal incidence $P(\mathbf{Q})$ is a symmetric function, so that its Fourier transform is real. The probability density $f_{Z}(z ; \mathbf{R})$ can now be found if one recalls that:

$$
\left\langle e^{i k_{z} Z(\mathbf{R})}\right\rangle=\int d z e^{i k_{z} z} f_{Z}(z ; \mathbf{R}) .
$$

Formally, therefore, $f_{Z}(z ; \mathbf{R})$ is fully determined by the scattering intensity, through another Fourier transform:

$$
f_{Z}(z ; \mathbf{R})=\frac{A}{(2 \pi)^{2}} \int d k_{z} e^{-i k_{z} z} \mathcal{F}_{k_{z}}^{-1}[P(\mathbf{Q}) ; \mathbf{R}] .
$$

The last equation shows that He scattering may in principle be used to fully invert the probability density of the corrugation function of a randomly corrugated surface. However, this requires dense sampling of the scattering intensities over a broad range of incidence wave-numbers, a task which may be difficult to accomplish in practice. Additional difficulties may arise due to Gibbs phenomenon and noise. A more realistic approach is to determine moments of the random variable describing the surface corrugation, an idea which dates back to the Backus and Gilbert work in geophysics [38], and has been pursued by others as well [26, 39]. To show how this may be accomplished here one may expand the characteristic function in terms of its moments. We then obtain from Eqs.(10), (11):

$$
\sum_{n=0}^{\infty} \frac{\left(i k_{z}\right)^{n}}{n !}\left\langle Z(\mathbf{R})^{n}\right\rangle=\frac{A}{2 \pi} \mathcal{F}_{k_{z}}^{-1}[P(\mathbf{Q}) ; \mathbf{R}] \equiv g\left(k_{z} ; \mathbf{R}\right)
$$

The moments of $Z(\mathbf{R})$ may now be found by differentiation of the experimentally available function $g\left(k_{z} ; \mathbf{R}\right)$ :

$$
\left\langle Z(\mathbf{R})^{n}\right\rangle=\frac{1}{i^{n}} \frac{d^{n} g(0 ; \mathbf{R})}{d k_{z}^{n}} .
$$

Thus in contrast to the rather involved task of extraction of the full probability density via Eq. (12), requiring an experiment to be performed over the entire energy range, extraction of the moments merely involves an extrapolation of the data to the low energy limit $k_{z}=0$. Progressively higher moments, however, require higher derivatives and hence an increasingly dense sampling in $k_{z}$ to reduce noise due to finite differences. As a check, one may estimate the first moment $\langle Z(\mathbf{R})\rangle=2\langle\xi(0)-\xi(\mathbf{R})\rangle$, which must clearly vanish. In practice, perhaps the most interesting piece of information is the second moment. Assuming that all higher moments vanish is equivalent to the assumption that $Z(\mathbf{R})$ is a Gaussian random variable. From Eqs.(13), 114) one has:

$$
\left\langle Z(\mathbf{R})^{2}\right\rangle=-\left.\frac{A}{2 \pi} \frac{d^{2}}{d k_{z}^{2}}\left[\mathcal{F}_{k_{z}}^{-1}[P(\mathbf{Q}) ; \mathbf{R}]\right]\right|_{k_{z}=0} .
$$

One may also choose to focus on the corrugation function $\xi(\mathbf{R})$ itself, instead of on the relative corrugation $Z(\mathbf{R})=$ $2[\xi(0)-\xi(\mathbf{R})]$. Without loss of generality one can take $\langle\xi(0)\rangle=\langle\xi(\mathbf{R})\rangle=0$. A cumulant expansion can then be used to evaluate the averages in Eq.(9). Truncating this expansion at the second cumulant, or equivalently, assuming that $\xi(\mathbf{R})$ is a Gaussian random variable, one obtains:

$$
P(\mathbf{Q})=\frac{1}{A} \int d \mathbf{R} e^{i \mathbf{Q} \cdot \mathbf{R}} e^{\left(2 \sigma k_{z}\right)^{2}[C(\mathbf{R})-1]},
$$

where the variance and correlation function are respectively

$$
\sigma^{2}=\left\langle\xi(\mathbf{R})^{2}\right\rangle, \quad C(\mathbf{R})=\frac{1}{\sigma^{2}}\langle\xi(0) \xi(\mathbf{R})\rangle
$$

Here, by definition, $0 \leq|C(\mathbf{R})| \leq 1$ and $C(\mathbf{R}) \rightarrow 0$ as $R \rightarrow \infty$. Fourier transforming the scattering intensity now yields an expression which can be used to conveniently fit $\sigma^{2}$ and $C(\mathbf{R})$ as a function of $k_{z}$ :

$$
e^{\left(2 \sigma k_{z}\right)^{2}[C(\mathbf{R})-1]}=\frac{A}{2 \pi} \mathcal{F}_{k_{z}}^{-1}[P(\mathbf{Q}) ; \mathbf{R}] .
$$

A set of equations [notably Eqs.(12), (15), (18)] has thus been derived which can be applied to extract useful statistical information on the surface corrugation, within a hard wall model, from the angular intensity distribution. In the next section I will consider what information can be derived within the more realistic square well model. 


\section{Correlation Functions in the Square Well Model}

It is not possible to analytically solve for the probability density of the effective surface corrugation or its moments in the square well model, as was done in Sec.IIIB in the hard wall case. It is, however, possible to obtain some interesting information by performing a cumulant expansion, as I now proceed to show. An inverse Fourier transform of the scattering intensity in the general case of a translationally invariant potential [Eq.(6)] yields [using Eq.(7))]:

$$
\left\langle e^{i E(\mathbf{R})}\right\rangle=\frac{A}{2 \pi} \mathcal{F}^{-1}[P(\mathbf{Q}) ; \mathbf{R}] .
$$

Introducing the notation

$$
\Delta Z(\mathbf{R})=2[\Delta \xi(\mathbf{R})-\Delta \xi(0)]
$$

one obtains with the help of Eq.(8), after some algebra:

$$
E(\mathbf{R}) \approx k_{z}\left[Z(\mathbf{R})-\frac{1}{2} \delta \Delta Z(\mathbf{R})\right]
$$

This expression is exact to first order in the small parameter $\delta$. Without loss of generality again set $\langle Z(\mathbf{R})\rangle=0$, and also $\langle\Delta Z(\mathbf{R})\rangle=0$, so that the first moment of $E(\mathbf{R})$ vanishes. For the second moment one obtains, again to first order in $\delta$ :

$$
\left\langle E(\mathbf{R})^{2}\right\rangle-\langle E(\mathbf{R})\rangle^{2} \approx k_{z}^{2}\left[\left\langle Z(\mathbf{R})^{2}\right\rangle-\delta\langle Z(\mathbf{R}) \Delta Z(\mathbf{R})\rangle\right]
$$

In analogy to Eq.(17) define a variance and correlation function between the hard wall corrugation and the deviation from it due to the attractive well:

$$
\sigma_{1}^{2}=\langle\xi(\mathbf{R}) \Delta \xi(\mathbf{R})\rangle, \quad C_{1}(\mathbf{R})=\frac{1}{\sigma_{1}^{2}}\langle\xi(\mathbf{R}) \Delta \xi(0)\rangle,
$$

so that

$$
\langle Z(\mathbf{R}) \Delta Z(\mathbf{R})\rangle=8 \sigma_{1}^{2}\left[1-C_{1}(\mathbf{R})\right] .
$$

Collecting the results we obtain finally to second order in the cumulant expansion:

$$
e^{\left(2 \sigma k_{z}\right)^{2}[C(\mathbf{R})-1]-\left(2 \sigma_{1} \epsilon\right)^{2}\left[C_{1}(\mathbf{R})-1\right]}=\frac{A}{2 \pi} \mathcal{F}^{-1}[P(\mathbf{Q}) ; \mathbf{R}] .
$$

This equation [compare to Eq. (18)] may be used to fit the experimental data to obtain the correlation functions, variances, and the well depth of the attractive part of the potential. This is much information to fit to a single experimental function. However, since the contribution due to the attractive well is expected to be small, one may at first neglect this contribution. This is tantamount to assuming a hard wall interaction, by which one may obtain an estimate of the hard wall quantities $\sigma$ and $C(\mathbf{R})$. Having computed these, one may correct the fit by inclusion of $\sigma_{1}$, $C_{1}(\mathbf{R})$, and $\epsilon$, and proceed iteratively. In the next section I will consider how statistical information can be derived from the experimentally straightforward measurement of the specular intensity.

\section{Extraction of the Correlation Length from Specular Peak Measurements in the Hard Wall Model}

From an experimental point of view, there is a significant advantage to working with specular intensities, as their measurement involves a relatively minor effort compared to that required to obtain the full angular intensity distribution. There is also a theoretical advantage, in that energy transfer to the surface is significantly reduced in specular collisions, so that a phononless treatment is more justified. In the hard wall approximation, by addition and subtraction of the same term, Eq.(河) for the angular distribution can be written as

$$
\begin{aligned}
& P(\mathbf{Q})=\left|\frac{1}{A} \int d \mathbf{R} e^{i \mathbf{Q} \cdot \mathbf{R}}\left\langle e^{-2 i k_{z} \xi(\mathbf{R})}\right\rangle\right|^{2}+ \\
& \frac{1}{A^{2}} \int d \mathbf{R} \int d \mathbf{R}^{\prime} e^{i \mathbf{Q} \cdot\left(\mathbf{R}-\mathbf{R}^{\prime}\right)}\left[\left\langle e^{-2 i k_{z}\left[\xi(\mathbf{R})-\xi\left(\mathbf{R}^{\prime}\right)\right]}\right\rangle-\left\langle e^{-2 i k_{z} \xi(\mathbf{R})}\right\rangle\left\langle e^{2 i k_{z} \xi(\mathbf{R})}\right\rangle\right] .
\end{aligned}
$$


Assuming translational invariance again and specializing to the specular direction, one obtains with a second order cumulant expansion (see Ref. [33] for a more detailed derivation)

$$
I \equiv P(0)=e^{-\beta E}\left[1+\frac{1}{A} \int d \mathbf{R}\left(e^{\beta E C(\mathbf{R})}-1\right)\right],
$$

where for brevity:

$$
\beta=(2 \sigma)^{2}, \quad E=k_{z}^{2} .
$$

(It was not sufficient to simply set $\mathbf{Q}=0$ in Eq.(16) since then the integral would diverge due to the asymptotic properties of $C(\mathbf{R})$.)

I now wish to evaluate the specular intensity $I$ for some specific correlation functions. To be consistent with the truncation of the cumulant expansion at second order, I will investigate the case of short-range order, in which higher order moments of the corrugation function do not play a significant role. One is thus led to consider two types of short-range correlation functions. These are of course just convenient models, assumed for lack of further knowledge of the probability density distributions. In both cases I will be concerned with evaluating the integral term in Eq.(19), denoted by:

$$
F(E)=\frac{1}{2 \pi} \int d \mathbf{R}\left(e^{\beta E C(\mathbf{R})}-1\right)
$$

\section{Gaussian Correlation Function}

Let us assume a Gaussian form for the correlation function:

$$
C(R)=e^{-(R / l)^{2}}
$$

where $l$ is the correlation length. Then due to cylindrical symmetry

$$
F(E)=\int_{0}^{L}\left[e^{\beta E\left(e^{-(R / l)^{2}}-1\right)}-1\right] R d R
$$

where $L$ is the linear extent of the surface. As such the integral cannot be evaluated analytically, but its derivative with respect to the energy can:

$$
\frac{\partial F}{\partial E}=\frac{l^{2}}{2 E}\left(e^{\beta E}-e^{\beta E e^{-(L / l)^{2}}}\right) .
$$

Since short-range order was assumed one may safely neglect $\exp \left(-(L / l)^{2}\right)$. Differentiating Eq.(19) and combining with Eq. 20) then yields:

$$
\frac{\partial I}{\partial E}+\beta I+\frac{\pi l^{2}}{A} \frac{e^{-\beta E}-1}{E}=0
$$

This equation can be used conveniently for a best-fit of the correlation length $l$ and the variance $\sigma^{2}=\beta / 4$, by using the experimental specular data, as a function of incidence energy $E=k_{z}^{2}$.

\section{Exponential Correlation Function}

Next assume a longer range, exponential form for the correlation function:

$$
C(R)=e^{-(R / l)},
$$

where $l$ is a new correlation length. Then:

$$
F(E)=\int_{0}^{L}\left[e^{\beta E\left(e^{-(R / l)}-1\right)}-1\right] R d R .
$$


Again the integral cannot be evaluated analytically, but its derivative can:

$$
\frac{\partial F}{\partial E}=\frac{l^{2}}{E}[\operatorname{Ei}(\beta E)-\log (\beta E)-\gamma] .
$$

[neglecting $\exp (-L / l)]$. Here $\operatorname{Ei}(x)=e^{x}\left(1 / x+\int_{0}^{\infty} e^{-t}(x-t)^{-2} d t\right)$ is the exponential integral [40], and $\gamma$ is Euler's constant. Differentiation of Eq.(19) in combination with Eq.(21) now yields:

$$
\frac{\partial I}{\partial E}+\beta I+\frac{2 \pi l^{2}}{A E} e^{-\beta E}[\gamma+\log (\beta E)-\operatorname{Ei}(\beta E)]=0,
$$

which can be used to best-fit the correlation length and the variance for a model of exponentially decaying correlations.

\section{SUMMARY AND CONCLUSIONS}

This paper has presented new results on the inversion of structure of randomly corrugated surfaces from atom scattering data, within the SA. This work has been largely formal, with applications to be worked out in the future in connection with presently unavailable experimental data. The analysis presented here showed that within the framework of the SA, the scattering intensities in principle contain the full statistical information characterizing the surface disorder. Several potentially useful expressions were derived from which statistical parameters can be extracted in simple He scattering experiments. One application of the theory presented here is contained in our work on scattering from $\mathrm{Ag} / \mathrm{Pt}(111)$ [13], in which it was shown how randomly distributed adsorbates affect the scattering intensities. Additional theoretical applications will be undertaken in the future, but it is hoped above all that this work will stimulate experimentalists to further utilize inert atom scattering in the study of increasingly complex surface disorder. The results presented here suggest that such experiments can reveal a wealth of information concerning disordered surface structure, in particular on the statistics of randomly corrugated surfaces.

\section{ACKNOWLEDGEMENTS}

This work was carried out while the author was with the Physics Department and the Fritz Haber Center for Molecular Dynamics at the Hebrew University of Jerusalem, Givat Ram, Jerusalem 91904, Israel. Numerous helpful discussions with Prof. R. Benny Gerber, without whom this work could not have been completed, are gratefully acknowledged. Partial support from NSF Grant CHE 97-32758 is gratefully acknowledged as well.

[1] T. Engel and K.H. Rieder, in Structural Studies of Surfaces, Vol. 91 of Springer Tracts in Modern Physics, edited by G. Höler and E.A. Niekisch (Springer, Berlin, 1982), p. 55; B. Poelsema and G. Comsa In Springer Tracts in Modern Physics, Vol. 115, Scattering of Thermal Energy Atoms from Disordered Surfaces (Springer Verlag, Berlin, 1989); Proceedings of the Int. Conference on Molecule-Surface Interactions, edited by H. Ibach (North-Holland, Jülich, Germany, 1991); Helium Atom Scattering from Surfaces, edited by E. Hulpke (Springer-Verlag, Heidelberg, 1992); K.H. Rieder, Surf. Rev. \& Lett. 1, 51 (1994).

[2] R.B. Gerber, Molecular Scattering from Surfaces: Theoretical Methods and Results, Chem. Rev. 87, 29 (1987); R.B. Gerber, Theory of atom scattering from ordered and from disordered surfaces, in Dynamical Processes in Molecular Physics, edited by Delgado-Bario (IOP, Bristol, 1993), p. 299.

[3] Helium Scattering from Disordered Surfaces: (I) Theory and Methodology; (II) Applications to Some Fundamental Types of Disorder, D.A. Lidar (Hamburger) and R.B. Gerber, to be published in Comm. on Atomic and Mol. Phys.

[4] R.B. Gerber and A.T. Yinnon, Approximate solution of the inversion problem for atom-surface scattering, J. Chem. Phys. 73, $3232(1980)$.

[5] T.-S. Ho and H. Rabitz, Inversion of gas-surface scattering data for potential determination using functional sensitivity analysis. I. A case study for the He-Xe/C(0001) potential J. Chem. Phys. 94, 2305 (1991).

[6] D.A. Hamburger and R.B. Gerber, Optical theorem and the inversion of cross section data for atom scattering from defects on surfaces, J. Chem. Phys. 102, 6919 (1995), LANL preprint cond-mat/9512003. 
[7] N. Esbjerg and J.K. Norskov, Dependence of the He-scattering potential at surfaces on the surface-electron-density profile, Phys. Rev. Lett. 45, 807 (1980); N. D. Lang and J.K. Norskov, Interaction of helium with a metal surface, Phys. Rev. B 27, 4612 (1983).

[8] R.B. Laughlin, Helium diffraction from the GaAs(110) surface and the generation of helium-surface potentials, Phys. Rev. B 25, 2222 (1982); A. Sakai, M.J. Cardillo and D.R. Hamann, He-Si(100) potential: charge superposition and model structures, Phys. Rev. B 33, 5774 (1986); M.B. Nardelli, A density-functional study of van der Waals forces: He interaction with a semiconductor surface, Solid State Commun. 97, 215 (1996).

[9] N. Garcia, A. Barker and I.P. Batra, Crystallographic determination of metal surfaces with helium scattering, J. Elect. Spect. \& Related Phenomena 30, 137 (1983); D. Gorse, B. Salanon, F. Fabre and A. Kara, Diffraction of helium from $\mathrm{Cu}(110),(113),(115)$ and (117): interaction potential and surface crystallography, Surf. Sci. 147, 611 (1984); H. Jonsson and J.H. Weare, Low energy He atom scattering from $\mathrm{Ag}(110)$ and $\mathrm{Ag}(111)$ : is there an effective two-body potential?, Surf. Sci. 181, 495 (1987); F. Gogtas, The study of He scattering from Cu(110) with a new model potential, Mod. Phys. Lett. B 5, 1795 (1991); P. Cortona, M.G. Dondi, A. Lausi and F. Tommasini, Electron density and structure of the $(1 \times 2)-\mathrm{Au}(110)$ surface studied by He-beam scattering, Surf. Sci. 276, 333 (1992).

[10] R.W. Godby and N. Garcia, Breakdown of atomic superposition for the helium scattering potential of $\mathrm{c}(2 * 2)$ oxygen on nickel (001), Surf. Sci. 163, L681 (1985).

[11] C. Schwartz, Calculations of helium diffraction intensities from clean and hydrogen-plated platinum, Surf. Sci. 173, 503 (1986).

[12] C. Ramseyer, C. Girardet, P. Zeppenfeld, J. Goerge and G. Comsa, Xe monolayer adsorption on Cu(110): experiments and interaction calculations, Surf. Sci. 313, 251 (1994).

[13] A.T. Yinnon, D.A. Lidar (Hamburger), I. Farbman, R.B. Gerber, P. Zeppenfeld, M.A. Krzyzowski and G. Comsa, He scattering from random adsorbates, disordered compact islands, and fractal submonolayers: intensity manifestations of surface disorder, J. Chem. Phys. 106, 4228 (1997), LANL preprint quant-ph/9611038.

[14] G. Vidali, G. Ihm, H.-Y Kim and M.W. Cole, Potentials of Physical Adsorption, Surf. Sci. Rep. 12, 133 (1991).

[15] A.T. Yinnon, E. Kolodney, A. Amirav, R.B. Gerber, Atom-surface potentials by inversion of diffraction data: application to $\mathrm{He} / \mathrm{MgO}(100)$, Chem. Phys. Lett. 123, 268 (1986).

[16] W.A. Schlup, On the inversion of the atomic beam scattering data in the Eikonal approximation, Surf. Sci. 211, 180 (1989).

[17] R. Viswanathan, S. Shi, E. Vilallonga and H. Rabitz, Sensitivity of elastic gas-surface scattering to the potential: a functional sensitivity approach based on wave packet dynamics, J. Chem. Phys. 92, 3170 (1990).

[18] T.-S. Ho, H. Rabitz, Inversion of gas-surface scattering data for potential determination using functional sensitivity analysis. II. Extraction of the full interaction potential from low energy diffraction data, J. Chem. Phys. 96, 7092 (1992).

[19] J.M. Hutson and C. Schwartz, Selective adsorption resonance in the scattering of helium atoms from xenon coated graphite: close-coupling calculations and potential dependence, J. Chem. Phys. 79, 5179 (1983).

[20] A.T. Yinnon, D.A. Lidar (Hamburger), R.B. Gerber, P. Zeppenfeld, M.A. Krzyzowski and G. Comsa, Structure Determination of Disordered Metallic Sub-Monolayers by Helium Scattering: A Theoretical and Experimental Study, Surf. Sci. Lett, in press.

[21] R.B Gerber, A.T. Yinnon and J.N. Murrel, Sudden decoupling approximations for atom-surface scattering, Chem. Phys. 31, 1 (1978); J.I. Gersten, R.B. Gerber, D.K Dacol and H. Rabitz, Scattering from disordered surfaces in the sudden approximation, J. Chem. Phys. 78, 4277 (1983).

[22] A.T. Yinnon, S. Bosanac, R.B Gerber and J.N. Murrel, Coupled-channel calculations and the accuracy of the sudden approximation for atom-surface scattering, Chem. Phys. Lett. 58, 364 (1978).

[23] R.B. Gerber, A.T. Yinnon and R. Kosloff, Effects of isolated impurities on atom scattering from crystalline surfaces: exact quantum-mechanical calculations, Chem. Phys. Lett. 105, 523 (1984).

[24] Atom Scattering from Disordered Surfaces in the Sudden Approximation: Double Collisions Effects and Quantum Liquids, D.A. Lidar (Hamburger), Surf. Sci., in press.

[25] L.E. Reichl, A Modern Course in Statistical Physics (University of Texas Press, Austin, 1980).

[26] K. Chadan and P.C. Sabatier, Inverse problems in quantum scattering theory (Springer-Verlag, New York, 1989).

[27] M.V. Klibanov and P.E. Sacks, Phaseless inverse scattering and the phase problem in optics, J. Math. Phys. 33, 3813 (1992); M.V. Klibanov, P.E. Sacks and A.V. Tikhonravov, The phase retrieval problem, Inverse Problems 11, 1 (1995).

[28] V.O. De Haan, A.A. van Well, P.E. Sacks, S. Adenwalla and others, Toward the solution of the inverse problem in neutron reflectometry, Physica B 221, 524 (1996).

[29] J.L. Beeby, The scattering of helium atoms from surfaces, J. Phys. C 4, L359 (1971); J.L. Beeby, Quantum mechanical scattering of atoms from surfaces, Comm. on Solid State Phys. 7, 1 (1975).

[30] R. von Mises, Mathematical Theory of Probability and Statistics (Academic Press, New York, 1964).

[31] N. Flytzanis and V. Celli, Model calculations of atom-surface scattering, Surf. Sci. 42, 173 (1974); N. Garcia, G. Armand and J. Lapujoulade, Diffraction intensities in helium scattering; topographic curves, Surf. Sci. 68, 399 (1977); F.O. Goodman, Scattering of atoms by a stationary sinusoidal hard wall: rigorous treatment in $(n+1)$ dimensions and comparison with the Rayleigh method, J. Chem. Phys. 66, 976 (1977); F.O. Goodman, Theory of scattering of He by a W(112) surface, Surf. Sci. 70, 578 (1978); J. Harris, A. Liebsch, G. Comsa and G. Mechtersheimer, Refraction effects in atom scattering from stepped surfaces, Surf. Sci. 118, 279 (1982); A.C. Levi, G. Benedek, L. Miglio and G. Platero, Surface Green function 
matching approach to the surface dynamics ofionic crystals. II. Theoretical analysis of the inelastic scattering of He from $\mathrm{NaF}(001)$ in the eikonal approximation, Surf. Sci. 143, 253 (1984);

[32] J. Harris and A. Liebsch, Theoretical aspects of helium scattering from metal surfaces, Vacuum 33, 655 (1983).

[33] D.K Dacol, H. Rabitz and R.B. Gerber, Atom scattering from disordered surfaces: Randomly corrugated hard walls and the sudden approximation, J. Chem. Phys. 86, 1616 (1987).

[34] M. Born, Principles of Optics, 6 ed. (Pergamon Press, Oxford, 1980).

[35] J.H. Weare, Helium scattering from solid surfaces, J. Chem. Phys. 61, 2900 (1974).

[36] N. Garcia, Normalized distorted-wave Born approximation for soft potentials in atom-surface scattering, Phys. Rev. B 25, 4994 (1982).

[37] G. Armand and J.R. Manson, An exact iterative solution of the atom-surface scattering problem for realistic potentials, Surf. Sci. 119, L299 (1982).

[38] G. Backus and F. Gilbert, Geophys. J. R. Astron. Soc. 13, 247 (1967).

[39] A.K. Louis, Approximate inverse for linear and some nonlinear problems, Inverse Problems 12, 175 (1996).

[40] I.S. Gradshteyn, I.M. Ryzhik, Table of Integrals, Series and Products (Academic Press, New York, 1980). 